42.

\section{Paramedic Evaluation of Clinical Indicators of Cervical Spine Injury}

Ritu Sahni, MD, * James J. Menegazzi, PhD, Vincent N. Mosesso, MD Department of Emergency Medicine, University of Pittsburgh, Pittsburgh, Pennsylvania USA

Purpose: Standard prehospital practice includes frequent immobilization of blunt trauma patients, some based solely on mechanism. Unnecessary cervical spine (c-spine) immobilization does have disadvantages, including morbidity such as low back pain and splinting, increased scene time and costs, and patient-paramedic conflict. Emergency physicians (EPs) use clinical criteria to clear trauma patients of c-spine injury. If paramedics were able to apply clinical criteria in the out-of-hospital setting, then unnecessary c-spine immobilization could be safely avoided. We designed a prospective, randomized, simulated trial to determine the level of agreement between paramedic and EP assessment of clinical indicators of cervical spine injury. We hypothesized that there would be substantial agreement between paramedic and EP evaluation of standardized patients.

Methods: A convenience sample of ten paramedics and ten attending EPs participated. Ten standardized patients, with various combinations of positive and negative findings, were examined simultaneously by EP-paramedic pairs. Each pair evaluated five randomly assigned patients for six clinical criteria, which were: 1) alteration in consciousness, 2) evidence of intoxication, 3) complaint of neck pain, 4) cervical tenderness, 5) neurologic deficit or complaint, and 6) distracting injury. If any criterion was positive, that was considered an immobilization decision. The kappa statistic was utilized to determine level of agreement between the two groups for each individual criterion and for the immobilization decision. A kappa of 0.40 to 0.75 denotes good reproducibility and $>0.75$ denotes excellent reproducibility.

Results: Kappa for each criterion was: 1) 0.77, 2) $0.68,3) 0.62$, 4) $0.74,5) 0.68$, and 6)

0.62. The kappa statistic for immobilization decision was 0.90 . In only one case did the immobilization decision differ; the paramedic indicated immobilization whereas the physician did not.

Conclusion: In our model, there was excellent agreement between paramedics and physicians when evaluating simulated patients for possible c-spine injury. No patient requiring immobilization would have been clinically cleared by paramedics. These data support the next step; a prospective field trial evaluating the use of these criteria by paramedics.
45.

The Impact of an Alert Tone on On-Scene Time in Trauma Patients

Todd J. Le Duc, BA, NREMT-P, * William E. Goellner, MD, FACEP, Nabil E. Sanadi, $M D, M B A, F A C E P$

Broward County Fire Rescue Division, Broward General Medical Center, Ft. Lauderdale, Florida USA

Objective: Determine whether radio alerts to paramedics after 7 minutes of on-scene time reduces total on-scene time for trauma patients.

Methods: Paramedics radio base once they determine ACS criteria were met. Paramedics were informed by radio when 7 minutes lapsed on-scene. Dispatch times were recorded.

Results: The control group (Nov. 1-30, 1995) of 135 consecutive patients were reviewed without a radio alert. The test group (Dec. 1-31, 1995) of 103 consecutive patients, with a radio alert message 7 minutes after scene arrival were also reviewed. Groups were matched for extrication times, blunt versus penetrating trauma, age, etc. We analyzed both groups for the interval of time from announcement of "trauma alert," (when paramedics determined the patient met ACS criteria or from the time extrication was complete (if applicable) to the time en route to the hospital. Average on-scene time for the control group was 13.7 minutes [range 4-35] versus test group 9.3 minutes [range 2-26] $(p<0.001)$.

Conclusion: A 7 minute alert significantly shortens on-scene time ( 4.4 minutes). This allows patients to receive definitive therapy earlier. Further evaluation is needed to determine whether clinical outcomes are affected by shorter on-scene times. 